\title{
Association between Mental Health and Arterial Stiffness in Middle-aged and Older Adults
}

\author{
Nobuhiko Akazawa ${ }^{\dagger}$, Koichiro Tanahashi, Keisei Kosaki ${ }^{\S}$, Ai Hamasaki, Seiji Maeda*, \\ Department of Sports Medicine, Faculty of Health and Sport Sciences, University of Tsukuba, 1-1-1 Tennodai, Taukuba, Ibaraki, Japan
}

\section{ARTICLE INFO}

\section{Article History}

Received 22 April 2020

Accepted 13 July 2020

Keywords

Pulse wave velocity

general health questionnaire

elderly

stress

\begin{abstract}
Background: Mental stress is a risk factor for cardiovascular disease. However, little is known about the impact of mental stress on arterial function in middle-aged and older adults.
\end{abstract}

Objective: This study is aimed to investigate the association between mental stress and arterial function in this population.

Methods: A total of 156 healthy middle-aged and older adults (aged 50-79 years) participated in this study. Mental stress was evaluated by the General Health Questionnaire (GHQ), and arterial stiffness was measured by the Carotid-femoral Pulse Wave Velocity (cfPWV).

Results: cfPWV was significantly correlated with the GHQ score, even after adjustment for age, sex, body mass index, and cholesterol, triglyceride, and glucose. Multiple regression analysis further indicated that GHQ score was a significantly independent determinant of cfPWV $(p<0.05)$.

Conclusion: GHQ is positively associated with arterial stiffness, independent of the potential confounding factors in healthy middle-aged and older adults.

(C) 2020 Association for Research into Arterial Structure and Physiology. Publishing services by Atlantis Press International B.V. This is an open access article distributed under the CC BY-NC 4.0 license (http://creativecommons.org/licenses/by-nc/4.0/).

\section{INTRODUCTION}

Cardiovascular Disease (CVD) is a major cause of morbidity and mortality globally. Age-related elevated blood pressure and metabolic-related biochemical markers including cholesterol, triglyceride, and glucose, are considered important conventional risk factors of CVD risk [1-3]. But, these traditional risk factors only do not fully account for the development in CVD events [4]. In addition to well-established risk factors, mental health status and psychological well-being has been also recognized as an important risk factor for CVD as well as behavioral concern about unhealthy diet, physical activity, and obesity [5,6]. Negative mental health may increase adrenal hormones, sympathetic nerve activity, and endothelial dysfunction, which all contributes to cardiovascular abnormality $[7,8]$. Prospective cohort studies have revealed that depression is likely to increase risk for the onset of CVD events $[9,10]$. Similarly, the status of general mental health, measured by self-reported screening, is

"Corresponding author. Email: maeda.seiji.gn@u.tsukuba.ac.jp

Present address:

${ }^{\dagger}$ Department of Sports Research, Japan Institute of Sports Sciences, 3-15-1 Nishigaoka, Kitaku, Tokyo, Japan

${ }^{\ddagger}$ Department of Health and Sports Sciences, Kyoto Pharmaceutical University, 5 MisasagiNakauchi-cho, Yamashina-ku, Kyoto, Kyoto, Japan

${ }^{s}$ Faculty of Sport Sciences, Waseda University, 2-579-15 Mikajima, Tokorozawa, Saitama, Japan

Peer review under responsibility of the Association for Research into Arterial Structure and Physiology

Data availability statement: The authors confirm that the data supporting the findings of this study are available within the article [and/or] its supplementary materials. also found to be a predictor of CVD [6,11]. Then, the relationship between psychological stress and normal functioning of the cardiovascular system seems to be have clinical and research interests.

A functional component of artery, large elastic artery stiffness such as the aorta and common carotid arteries, increases with advanced age [12]. Arterial stiffening leads to the loss of the ability to buffer and cushion oscillation in blood flow and blood pressure [13]. Increase in arterial stiffness contributes to elevations in systolic blood pressure, pulse pressure, left ventricular after load, and then is an independent risk factor for CVD [14]. Previous studies have demonstrated that arterial stiffness was associated with work-related stress in Japanese younger adults $[15,16]$. However, the impact of general mental health on arterial stiffness in older populations remains unclear. The aim of this study was to investigate whether mental health is associated with arterial stiffness. Therefore, the present study examined the association between mental stress and Pulse Wave Velocity (PWV) in healthy middle-aged and older adults.

\section{MATERIALS AND METHODS}

\subsection{Subjects}

We recruited the healthy and over 50 years subjects through local newspaper advertisements. We excluded the subjects treated with treatment for hypertension, dyslipidemia, or diabetes, and hormone 
replacement therapy. There were 162 eligible subjects in total. Six subjects were canceled for personal reason. A total of 156 subjects (men: $n=32$, women: $n=124$ ) were participated in this study. All subjects were non-smokers and free of cardiovascular disease. All women were postmenopausal, amenorrhea for at least 1 year. All potential risks associated with the study were explained to the subjects, and written informed consent was provided by all participants. All procedures were reviewed and approved by the Ethics Committee of the University of Tsukuba.

\subsection{Study Procedures}

All experiments were conducted in the morning after a 12-h overnight fast. Subjects abstained from alcohol and caffeine for at least $12 \mathrm{~h}$ and did not exercise for at least $24 \mathrm{~h}$ before the experiments. Measurements were performed in a quiet, temperature-controlled room (at $\left.24-26^{\circ} \mathrm{C}\right)$. We assessed the arterial stiffness, mental stress, and blood chemistries.

\subsection{Measurements}

\subsubsection{Arterial stiffness}

Carotid-femoral PWV (cfPWV) was measured as arterial stiffness by a semi-automated vascular access system, as previously described [17]. Briefly, carotid and femoral pressure waveforms were obtained by two applanation tomometry sensors incorporating an array of 15 transducers (Form PWV/ABI, Colin medical Technology, Komaki, Japan). The distance between the left common carotid and left femoral arterial recording sites was divided by the transit time resulting in the calculation of cfPWV. Simultaneously, brachial systolic blood pressure and diastolic blood pressure, and heart rate were assessed by an electrocardiogram and an oscillometric extremity cuff.

\subsubsection{Mental stress}

Mental stress was assessed by self-reported survey, 60 item General Health Questionnaire (GHQ) [18]. This is suitable for the screening of psychological well-being, including psychological distress, depression, anxiety, and social impairment, and so on [19]. Each symptom was rated on Likert scale of frequency from 1 to 5 , to maximize the response variability. Higher scores indicated higher levels of psychological distress.

\subsubsection{Blood chemistries}

Blood samples were collected from the antecubital vein after overnight fasting. Plasma and serum were collected and stored at $-80^{\circ} \mathrm{C}$, until the assay was performed. Serum cortisol concentration was determined using the chemiluminescence immunoassay method, serum total cholesterol, triglyceride, and plasma glucose were determined using the standard enzymatic technique, all by a commercial laboratory (LSI Medience Corporation Ltd., Tokyo, Japan).

\subsection{Statistical Analyses}

All data characteristics are expressed as means \pm Standard Deviation (SD). Spearman's rank correlation coefficient was used to determine the association between cfPWV and partial correlation analyses adjusted for age, sex, and biochemistries, which showed significant association with cfPWV in the simple correlation analysis, for example triglyceride, glucose and cortisol. Multiple regression analysis was performed to determine the independent correlation between cfPWV and variables, and those covariates were entered as dependent variables in the subsequent multiple linear regression analysis. Statistical data analyses were performed using the SPSS software (Version 24; IBM, Armonk, NY, USA), and statistical significance was set a priori at $p<0.05$ a priori, for all comparisons.

\section{RESULTS}

The characteristics of the participants are presented in Table 1. Table 2 illustrates the simple correlation of cfPWV with cardiovascular risk factors and mental stress variables. cfPWV was significantly correlated with age, sex, triglycerides, glucose, cortisol, and the GHQ score $(\rho=0.340,0.320,0.192,0.314,0.215$ and 0.163 , respectively, all $p<0.05)$. The significant correlation between cfPWV and the GHQ score was evident after adjustment for age,

Table $1 \mid$ The characteristics of the study participants

\begin{tabular}{lc}
\hline Variables & \\
\hline Age (years) & $62 \pm 6$ \\
Height $(\mathrm{cm})$ & $159 \pm 7$ \\
Weight $(\mathrm{kg})$ & $57 \pm 9$ \\
Body mass index $\left(\mathrm{kg} / \mathrm{m}^{2}\right)$ & $23 \pm 3$ \\
Total cholesterol (mg/dL) & $224 \pm 35$ \\
Triglyceride $(\mathrm{mg} / \mathrm{dL})$ & $98 \pm 53$ \\
Glucose $(\mathrm{mg} / \mathrm{dL})$ & $94 \pm 10$ \\
Cortisol $(\mu \mathrm{g} / \mathrm{dL})$ & $8 \pm 3$ \\
GHQ score $(\mathrm{points})$ & $45 \pm 16$ \\
Heart rate $(\mathrm{bpm})$ & $61 \pm 7$ \\
Systolic blood pressure (mmHg) & $125 \pm 15$ \\
Diastolic blood pressure $(\mathrm{mmHg})$ & $75 \pm 9$ \\
Mean arterial blood pressure & $94 \pm 12$ \\
$\quad(\mathrm{mmHg})$ & $925 \pm 155$ \\
cfPWV $(\mathrm{cm} / \mathrm{s})$ &
\end{tabular}

Table 2 The correlation coefficients of carotid-femoral pulse wave velocity with cardiovascular risk factor parameters and mental stress

\begin{tabular}{lcc}
\hline & \multicolumn{2}{c}{ Correlation to cfPWV } \\
\cline { 2 - 3 } Variables & Coefficient $\boldsymbol{r}$ & $\boldsymbol{p}$ \\
\hline Age (years) & 0.340 & $<0.001$ \\
Sex & 0.320 & $<0.001$ \\
Body mass index $\left(\mathrm{kg} / \mathrm{m}^{2}\right)$ & 0.027 & 0.747 \\
Total cholesterol $(\mathrm{mg} / \mathrm{dL})$ & 0.140 & 0.092 \\
Triglyceride $(\mathrm{mg} / \mathrm{dL})$ & 0.192 & $<0.05$ \\
Glucose $(\mathrm{mg} / \mathrm{dL})$ & 0.314 & $<0.001$ \\
Cortisol $(\mu \mathrm{g} / \mathrm{dL})$ & 0.215 & $<0.05$ \\
GHQ score $(\mathrm{points})$ & 0.163 & $<0.05$ \\
\hline
\end{tabular}

$r$ : Spearman's rank correlation coefficient. 
Table 3 Multiple regressions on arterial stiffness

\begin{tabular}{|c|c|c|c|c|c|c|c|}
\hline \multirow{2}{*}{ Variables } & \multirow{2}{*}{$B$} & \multirow{2}{*}{ SEB } & \multirow{2}{*}{$\beta$} & \multirow{2}{*}{$r$} & \multirow{2}{*}{$p$} & \multicolumn{2}{|c|}{$95 \%$ CI } \\
\hline & & & & & & Lower & Upper \\
\hline \multicolumn{8}{|c|}{$R^{2}=0.559(p<0.001)$} \\
\hline Age & 7.073 & 1.549 & 0.285 & 0.354 & $<0.001^{*}$ & 4.011 & 10.135 \\
\hline Sex & -63.167 & 24.517 & -0.164 & 0.285 & $0.011^{*}$ & -111.643 & -14.690 \\
\hline Body mass index & -4.399 & 3.437 & -0.083 & 0.036 & 0.203 & -11.196 & 2.397 \\
\hline MAP & 6.703 & 0.785 & 0.525 & 0.594 & $<0.001^{*}$ & 5.152 & 8.255 \\
\hline Total cholesterol & 0.167 & 0.282 & 0.037 & 0.129 & 0.554 & -0.390 & 0.725 \\
\hline Triglyceride & 0.323 & 0.181 & 0.114 & 0.232 & 0.077 & -0.035 & 0.681 \\
\hline Glucose & 0.971 & 0.934 & 0.065 & 0.299 & 0.301 & -0.876 & 2.817 \\
\hline Cortisol & 4.968 & 3.672 & 0.087 & 0.245 & 0.178 & -2.293 & 12.228 \\
\hline GHQ score & 1.772 & 0.555 & 0.189 & 0.164 & $0.002^{*}$ & 0.674 & 2.871 \\
\hline
\end{tabular}

indicates significance independent correlation. MAP, mean arterial blood pressure; CI, confidence interval.

sex, body mass index, cholesterol, triglyceride, glucose, and cortisol $(\rho=0.257, p<0.05)$; Table 3 shows the results of a multiple linear regression analysis for the association between cfPWV and independent variables including age, sex, body mass index, blood pressure, biochemistries, and mental stress. In addition to age, sex, and mean arterial blood pressure, the GHQ score was evidenced significantly as an independent determinant of $\operatorname{cfPWV}(\beta=0.189$, $p<0.05)$, but not for cortisol $(\beta=0.087, p=0.178)$.

\section{DISCUSSION}

This study showed a significant relationship between GHQ and cfPWV in healthy middle-aged and older Japanese individual. Multiple regression analysis demonstrated that age, sex, blood pressure, and the GHQ score were independently correlated with cfPWV. The results of the present analysis demonstrated that general mental health, in addition to traditional risk factors for CVD may mediate increase in arterial stiffness, which suggest that mental health-related increase in arterial stiffness involves in the development risk for CVD. Moreover, the GHQ score may be considered as predictor of pathophysiological deterioration of the future cardiovascular dysfunction [20].

Psychological distress contributes to development of hypertension and CVD. In addition, it has been demonstrated in an interventional study that acute mental stress increased arterial stiffness [21,22]. Researchers mainly reported that occupational and work-related stress, evaluated by the Job Content Questionnaire was inversely correlated with PWV in Japanese young adults [23]. However, Otsuka et al. [15] have demonstrated that work-related stress evaluated by the Brief Job Stress Questionnaire was positively correlated with wave reflection, as another index of arterial stiffness in young and middle-aged workers. The relationship between occupational stress and arterial stiffness may be in part affected by study subject and/or stress measurements. In this study, we firstly investigated the association between cfPWV and mental stress using the GHQ in healthy middle-aged and older adults, and found that cfPWV was also positively correlated with the GHQ score. General mental health status in habitual life may associated with the development of arterial stiffness in middle-aged and older adults.

The incident of CVD is generally attributed to the various factors, such as hypertension, biochemical and metabolic abnormalities, and smoking [24]. Psychological mental stress was also founded to be associated with increased risk of CVD [25]. More specifically, work-related mental stress was correlated with CVD, even in young and middle-aged populations [26]. The association between mental stress and CVD risk could be explained by stress-induced augmentation in increase in blood pressure in patients with myocardial infarction or abnormal lipid and glucose metabolism [27,28]. Otsuka et al. [15] have reported that deterioration of mental stress by work-related stress was correlated with arterial stiffness in middle-aged Japanese individual. We extended these observations to arterial stiffness in the healthy middle-aged older population who has retired from their job, and found that psychological stress assessed by the GHQ score was correlated with cfPWV. Furthermore, in this study, the significant correlation was evident after adjustment for age, sex and traditional risk factors for CVD, such as blood pressure, cholesterol, triglycerides, and glucose. Therefore, for healthy middle-aged and older adults, who have no hypertension, no lipid and glucose abnormalities, and are non-smokers, it is preferable to manage the mental health status rather than blood chemistries to preventing increase in arterial stiffness.

Physiological response to stressors induces the activation of the hypothalamic-pituitary-adrenal system and cortisol secreted from adrenal cortex, which is known as stress hormones. Cortisol is considered to be a potential mediator link between mental stress and CVD [29]. It has been reported that cortisol has detrimental effect on vascular tone, development, remodeling, and inflammation [30]. In addition, cortisol was involved in acute mental stress-induced endothelial dysfunction [31]. A cross-sectional study reported circulating level of cortisol was correlated with arterial stiffness measured by brachial-ankle PWV in young Japanese adults [32]. In this study, serum cortisol concentration and mental stress measured by the GHQ score were correlated with cfPWV. Moreover, the GHQ score was also significantly correlated with cfPWV after adjustment for confounders, whereas the correlation between serum cortisol concentration and cfPWV did not reach statistical significance. It is possible that self-reported habitual mental stress and cortisol response to stress independently affect arterial stiffness, evaluation of subjectively measurement of mental health are also more important for arterial deterioration in healthy individuals.

The present study has some limitations. First, the sample size was relatively small. We only recruited only healthy middle-aged and older adults aged over 50, because we tried to exclude other 
confounders such as hypertension, smoking, and metabolic syndrome. Second, the subjects in this study comprised mainly women. We observed the sex differences in cfPWV and the correlation between sex and cfPWV (Table 2). On the other hand, there was no significant differences in GHQ score between sexes. Additionally, the association between cfPWV and GHQ were significance after adjustment for sex and other confounders. However, the investigation on sex differences in the association between arterial stiffness and mental stress are necessary in the future. Third, this was a cross-sectional study that cannot establish a cause-effect relationship, though the results of the multivariate analyses indicate a statistically significant association between mental stress and arterial stiffness. Further prospective studies are warranted to determine whether the observed findings are clinically meaningful.

\section{CONCLUSION}

The primary finding of this study is that GHQ score is significantly associated with arterial stiffness, independent of the potential confounders such as age, sex, blood pressure, cholesterol, triglyceride, glucose, and cortisol. These results suggest that mental stress assessed by the GHQ may be a useful to subjective predictor of the risk of increase in arterial stiffness in healthy middle-aged and older adults.

\section{CONFLICTS OF INTEREST}

The authors declare they have no conflicts of interest.

\section{AUTHORS' CONTRIBUTION}

NA and SM contributed to conceptualization and funding acquisition. KT, KK and AH contributed to data curation and formal analysis. NA and SM contributed in writing (reviewed and edited).

\section{FUNDING}

This work was supported by The Japan Society for the Promotion of Science KAKENHI, Grand Number 16K16594.

\section{REFERENCES}

[1] Kitamura A, Sato S, Kiyama M, Imano H, Iso H, Okada T, et al. Trends in the incidence of coronary heart disease and stroke and their risk factors in Japan, 1964 to 2003: the Akita-Osaka study. J Am Coll Cardiol 2008;52:71-9.

[2] Levy D, Wilson PWF, Anderson KM, Castelli WP. Stratifying the patient at risk from coronary disease: new insights from the Framingham Heart Study. Am Heart J 1990;119:712-17.

[3] Chambless LE, Folsom AR, Sharrett AR, Sorlie P, Couper D, Szklo D, et al. Coronary heart disease risk prediction in the Atherosclerosis Risk in Communities (ARIC) study. J Clin Epidemiol 2003;56:880-90.
[4] Ueshima H. Explanation for the Japanese paradox: prevention of increase in coronary heart disease and reduction in stroke. J Atheroscler Thromb 2007;14:278-86.

[5] Greenwood DC, Muir KR, Packham CJ, Madeley RJ. Coronary heart disease: a review of the role of psychosocial stress and social support. J Public Health Med 1996;18:221-31.

[6] Hamer M, Molloy GJ, Stamatakis E. Psychological distress as a risk factor for cardiovascular events: pathophysiological and behavioral mechanisms. J Am Coll Cardiol 2008;52:2156-62.

[7] Iso H, Date C, Yamamoto A, Toyoshima H, Tanabe N, Kikuchi S, et al. Perceived mental stress and mortality from cardiovascular disease among Japanese men and women: the Japan Collaborative Cohort Study for Evaluation of Cancer Risk Sponsored by Monbusho (JACC Study). Circulation 2002;106:1229-36.

[8] Sun W, Schooling CM, Chan WM, Ho KS, Lam TH. The association between depressive symptoms and mortality among Chinese elderly: a Hong Kong cohort study. J Gerontol A Biol Sci Med Sci 2011;66:459-66.

[9] Chida Y, Steptoe A. Greater cardiovascular responses to laboratory mental stress are associated with poor subsequent cardiovascular risk status: a meta-analysis of prospective evidence. Hypertension 2010;55:1026-32.

[10] Van der Kooy K, van Hout H, Marwijk H, Marten H, Stehouwer C, Beekman A. Depression and the risk for cardiovascular diseases: systematic review and meta analysis. Int J Geriatr Psychiatry 2007;22:613-26.

[11] Robinson KL, McBeth J, Macfarlane GJ. Psychological distress and premature mortality in the general population: a prospective study. Ann Epidemiol 2004;14:467-72.

[12] Avolio AP, Deng FQ, Li WQ, Luo YF, Huang ZD, Xing LF, et al. Effects of aging on arterial distensibility in populations with high and low prevalence of hypertension: comparison between urban and rural communities in China. Circulation 1985;71:202-10.

[13] Nichols WW, O’Rourke MF, Vlachopoulos C. McDonald's Blood Flow in Arteries: Theoretical, Experimental, and Clinical Principles. 6th ed., London, UK: Hodder Arnold; 2011.

[14] Lakatta EG. Age-associated cardiovascular changes in health: impact on cardiovascular disease in older persons. Heart Fail Rev 2002;7:29-49.

[15] Otsuka T, Kawada T, Ibuki C, Kusama Y. Relationship between job strain and radial arterial wave reflection in middle-aged male workers. Prev Med 2009;49:260-4.

[16] Utsugi M, Saijo Y, Yoshioka E, Sato T, Horikawa N, Gong Y, et al. Relationship between two alternative occupational stress models and arterial stiffness: a cross-sectional study among Japanese workers. Int Arch Occup Environ Health 2009;82:175-83.

[17] Akazawa N, Ra SG, Sugawara J, Maeda S. Influence of aerobic exercise training on post-exercise responses of aortic pulse pressure and augmentation pressure in postmenopausal women. Front Physiol 2015;6:268.

[18] Nakagawa Y, Daibo E. Validity and reliability of the Japanese version of the General Health Questionnaire and its clinical application. Ichikawa, Japan: National Institute of Mental Health Japan; 1985 (in Japanese).

[19] Banks MH, Jackson PR. Unemployment and risk of minor psychiatric disorder in young people: cross-sectional and longitudinal evidence. Psychol Med 1982;12:789-98.

[20] Mattace-Raso FUS, van der Cammen TJM, Hofman A, van Popele NM, Bos ML, Schalekamp MADH, et al. Arterial stiffness 
and risk of coronary heart disease and stroke: the Rotterdam Study. Circulation 2006;113:657-63.

[21] Vlachopoulos C, Xaplanteris P, Alexopoulos N, Aznaouridis K, Vasiliadou C, Baou K, et al. Divergent effects of laughter and mental stress on arterial stiffness and central hemodynamics. Psychosom Med 2009;71:446-53.

[22] Tabara Y, Kohara K, Nakagawa S, Handa J, Hayashi M, Hamada C, et al. Effects of obesity and smoking on mental stress-induced blood pressure and augmentation index responses in normotensive young males: the J-SHIPP study. Hypertens Res 2008;31:1219-24.

[23] Nomura K, Nakao M, Karita K, Nishikitani M, Yano E. Association between work-related psychological stress and arterial stiffness measured by brachial-ankle pulse-wave velocity in young Japanese males from an information service company. Scand J Work Environ Health 2005;31:352-9.

[24] McGovern PG, Pankow JS, Shahar E, Doliszny KM, Folsom AR, Blackburn H, et al. Recent trends in acute coronary heart disease-mortality, morbidity, medical care, and risk factors. N Engl J Med 1996;334:884-90.

[25] Rosengren A, Hawken S, Ônpuu S, Sliwa K, Zubaid M, Almahmeed WA, et al. Association of psychosocial risk factors with risk of acute myocardial infarction in 11119 cases and 13648 controls from 52 countries (the INTERHEART study): case-control study. Lancet 2004;364:953-62.
[26] Aboa-Éboulé C, Brisson C, Maunsell E, Mâsse B, Bourbonnais R, Vézina $\mathrm{M}$, et al. Job strain and risk of acute recurrent coronary heart disease events. JAMA 2007;298:1652-60.

[27] Schnall PL, Schwartz JE, Landsbergis PA, Warren K, Pickering TG. Relation between job strain, alcohol, and ambulatory blood pressure. Hypertension 1992;19:488-94.

[28] Kawakami N, Araki S, Hayashi T, Masumoto T. Relationship between perceived job-stress and glycosylated hemoglobin in white-collar workers. Ind Health 1989;27:149-54.

[29] Koertge J, Al-Khalili F, Ahnve S, Janszky I, Svane B, SchenckGustafsson K. Cortisol and vital exhaustion in relation to significant coronary artery stenosis in middle-aged women with acute coronary syndrome. Psychoneuroendocrinology 2002;27:893-906.

[30] Walker BR. Glucocorticoids and cardiovascular disease. Eur J Endocrinol 2007;157:545-59.

[31] Broadley AJM, Korszun A, Abdelaal E, Moskvina V, Jones CJH, Nash GB, et al. Inhibition of cortisol production with metyrapone prevents mental stress-induced endothelial dysfunction and baroreflex impairment. J Am Coll Cardiol 2005;46:344-50.

[32] Nakao M, Nomura K, Karita K, Nishikitani M, Yano E. Relationship between brachial-ankle pulse wave velocity and heart rate variability in young Japanese men. Hypertens Res 2004;27:925-31. 